\title{
Nocturnal angina: precipitating factors in patients with coronary artery disease and those with variant angina
}

\author{
ARSHED A QUYYUMI, „ JOHN EFTHIMIOU, $†$ ANEES QUYYUMI, * \\ LORNA J MOCKUS, * STEPHEN G SPIRO, $\dagger$ KIM M FOX* \\ From the ${ }^{\star}$ National Heart Hospital and $\dagger$ University College Hospital, London
}

SUMMARY Factors precipitating noctural myocardial ischaemia were investigated in 10 patients with frequent daytime and nocturnal angina pectoris. Eight patients had fixed obstructive coronary artery disease or a low exercise threshold or both before the onset of ischaemia. Two patients had variant angina with normal coronary arteries and negative exercise tests. During sleep the electrocardiogram, electroencephalogram, electro-oculogram, electromyogram, chest wall movements, nasal airflow, and oxygen saturation were continuously measured. Forty two episodes of transient ST segment depression were recorded in the eight patients with coronary artery disease and 26 episodes of ST segment depression and elevation in the two patients with variant angina and normal coronary arteries. All episodes of ST segment depression in the former group of patients were preceded by an increase in heart rate as a result of arousal and lightening of sleep, bodily movements, rapid eye movement sleep, or sleep apnoea (one episode). In contrast, in the variant angina group no increase in heart rate, arousal, or apnoea preceded 23 of the 26 episodes of ST segment change. Thus increase in myocardial oxygen demand was important in precipitating noctural angina in patients with coronary artery disease and reduced coronary reserve. In the patients with coronary spasm these factors did not often precede the onset of noctural myocardial ischaemia.

Angina usually occurs on exertion during the day and is regarded as being secondary to an increase in myocardial oxygen demand that cannot be met by a simultaneous increase in blood flow because of obstructive coronary artery disease. It can also occur at rest and during sleep at night. The occurrence of nocturnal angina in patients with daytime pain was recognised in one of the earliest descriptions of the syndrome by Heberden. ${ }^{1}$ The suggestion that coronary artery spasm was a possible mechanism of angina pectoris, ${ }^{2}$ did not receive widespread support until Prinzmetal et al described the classic symptoms in patients with normal coronary arteries. ${ }^{3}$ Much more clinical, haemodynamic, and angiographic evidence has been accumulated in these patients that

Requests for reprints to Dr Kim M Fox, National Heart Hospital, Westmoreland Street, London W1M 8BA.

Accepted for publication 30 June 1986 supports coronary artery spasm as the underlying pathophysiological mechanism. ${ }^{45}$ It has been suggested, on the basis of these results, that even the nocturnal angina that occurs in patients with fixed obstructive coronary artery disease is secondary to coronary spasm or sudden reductions in coronary blood flow. 67

Episodes of ST segment depression and elevation, which may or may not be associated with chest pain, can be recorded by ambulatory electrocardiographic monitoring. ${ }^{8}$ In a preliminary study we examined the momentary changes in heart rate that preceded or accompanied episodes of nocturnal ST segment depression in patients with severe obstructive coronary artery disease. ${ }^{9}$ This detailed analysis showed that there was invariably a small but important increase in heart rate before the onset of ST segment depression in both painful and pain free nocturnal episodes. To evaluate further the mechanisms underlying nocturnal angina pectoris we have performed all night polygraphic studies in this group of 
patients and have compared their results with those of patients with normal coronary arteries and angiographically proven coronary spasm.

\section{Patients and methods}

\section{PATIENTS}

We studied six men and four women (aged 46 to 79 years) with frequent nocturnal and daytime angina pectoris that was associated with reversible ST segment changes. Patients $1-8$ had a very low exercise threshold before ST segment depression occurred (mean (SD) 2.9(1.4) min) during treadmill stress testing with a modified Bruce protocol. Severe three vessel coronary artery disease $(>70 \%$ coronary luminal narrowing) was demonstrable in five patients at angiography and at necropsy in one; one patient had a severe proximal left anterior descending artery stenosis only. The 79 year old patient did not have cardiac catheterisation. Patients 9 and 10 had a negative exercise test and no important coronary artery disease at angiography. Provocation with ergometrine maleate (mean dose $0.1 \mathrm{mg}$ ) during coronary angiography produced considerable localised spasm in a single coronary artery in both patients, which was accompanied by chest pain and ST segment elevation on the electrocardiogram.

\section{STUDY PROTOCOL}

Patients were weaned off all antianginal medication before the sleep studies. Glyceryl trinitrate was used to treat painful episodes. Polygraphic recordings were made during nocturnal sleep for one night in six patients and for two consecutive nights in four patients (mean (SD) 9(3) hours (range 6-13 hours)). Informed consent was obtained from all patients and the study was approved by the hospital ethics committee.

\section{POLYGRAPHIC RECORDINGS}

We obtained continuous overnight recordings of the electrocardiogram, electroencephalogram (C4/A1 or C3/A2 or both), electro-oculogram (two channels), and submental electromyogram. An ear oximeter (Hewlett Packard) was used to measure arterial oxygen saturation, nasal airflow was measured with a thermistor, and chest wall movements with an impedence pneumogram. Patients slept in a sound reduced room and were observed by one of us throughout each study. Recordings of all observations were obtained on an eight channel ink jet recorder (Siemens, Mingograph) which was run at $15 \mathrm{~mm} / \mathrm{s}$. Recordings of arterial oxygen saturation, chest wall movements, and nasal airflow were also obtained on a separate four channel paper recorder (Hewlett-Packard) run at $25 \mathrm{~mm} / \mathrm{min}$. Sleep was staged by the standard guidelines of Rechtschaffen and Kales. ${ }^{10}$

A continuous bipolar electrocardiogram of lead CM5 was obtained on a frequency modulated recorder (Oxford Medilog II) so that the ST segment shifts could be accurately measured. Important ST segment depression was defined as depression of $\geqslant 1 \mathrm{~mm}$ of the ST segment below the isoelectric line $0.08 \mathrm{~s}$ after the end of the QRS complex that persisted $\geqslant 1$ min. Important ST segment elevation was defined as a $\geqslant 1 \mathrm{~mm}$ shift upwards of the ST segment at the $\mathrm{J}$ point. Changes in the ST segment were measured with a magnifying lens equipped with a graticule, which enabled us to measure to an accuracy of $0.1 \mathrm{~mm}$. This meant that the exact time of onset of the ST segment change could be determined. ST segment shifts that occurred after changes in position were not regarded as important unless the changes returned to normal while the patients remained in the new position or if these episodes were followed by angina. For the purposes of this study isolated $T$ wave changes were not regarded as good indicators of myocardial ischaemia. Careful analysis of the R-R interval was performed for at least $5 \mathrm{~min}$ before the onset of ST segment change (baseline heart rate) and was continued throughout the episode. The $R-R$ interval was measured every $6 \mathrm{~s}$ to detect precisely the time of onset of any change in heart rate. Thus the exact time of onset of ST segment change and its relation to the change in heart rate could be determined. All the results are expressed as mean (SD).

\section{Results}

A total of 90 hours of recording was obtained during 14 nights of sleep in the 10 patients. The eight

Table Frequency and duration of episodes of ST segment change observed during sleep in patients with important coronary artery disease or positive exercise tests (cases 1-8) and patients with coronary spasm (cases 9 and 10)

\begin{tabular}{clllc}
\hline Cases & $\begin{array}{l}\text { Episodes of } S T \\
\text { depression }\end{array}$ & $\begin{array}{l}\text { Episodes of } S T \\
\text { elevation }\end{array}$ & $\begin{array}{l}\text { Number of painful } \\
\text { episodes }\end{array}$ & $\begin{array}{l}\text { Total duration of } \\
\text { episodes (min) }\end{array}$ \\
\hline $1-8$ & 42 & 0 & 18 & $64(56)$ (mean (SD)) \\
9 & 8 & 6 & 3 & 169 \\
10 & 11 & 1 & 2 & 167 \\
\hline
\end{tabular}




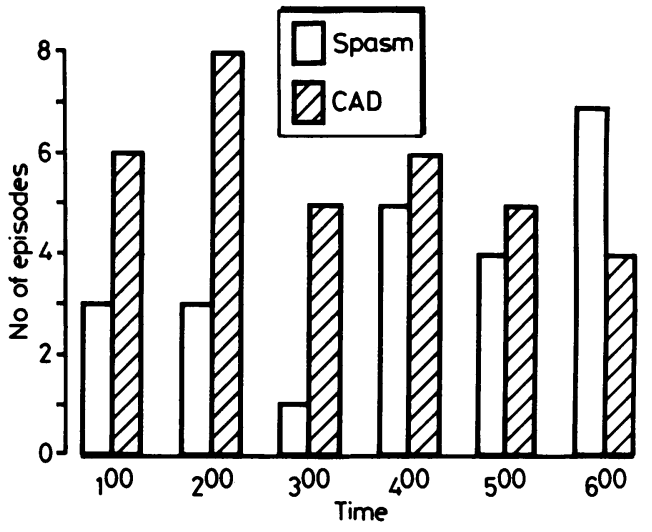

Fig 1 Hourly frequency of episodes of $S T$ segment change in patients with coronary artery disease (CAD) (hatched bars) and patients with normal coronary arteries and spasm (unhatched bars).

patients with obstructive coronary artery disease had 42 episodes of nocturnal ST segment depression (mean 5.3(3.5), range 1-13) of which $43 \%$ were painful, and they lasted from 1 to $81 \mathrm{~min}$ (table). In the two patients with normal coronary arteries and spasm there were 26 epsiodes of transient ST segment change during sleep, 19 with ST segment depression and seven with ST segment elevation. Of these, $19 \%$ were painful and they lasted from 2 to 8 min (table).

Episodes of ST segment change were equally distributed throughout the night, with no tendency for episodes to occur in the early morning hours in the eight patients (cases 1-8) with fixed obstructive coronary artery disease. The two patients with coronary spasm (cases 9 and 10) had more episodes of ST segment change lasting for a longer period in the early morning hours (16 episodes lasting $213 \mathrm{~min}$ from $4.00-7.00 \mathrm{am}$ ) compared with the early part of the night ( 7 episodes lasting $105 \mathrm{~min}$ from $1.00-4.00 \mathrm{am}$ ) (fig 1).

\section{RELATION BETWEEN ST SEGMENT CHANGES AND SLEEP STAGES}

Patients with coronary artery disease

In the patients (cases 1-8) with obstructive coronary artery disease and strongly positive stress tests most episodes of ST segment depression were preceded by arousal from deeper sleep stages to lighter sleep stages or to awakening; this was followed immediately by an abrupt increase in heart rate (fig 2). Often bodily movements, such as turning, followed the arousal and were associated with further increases in heart rate. Figure 2 shows an example of the onset of one episode of ST segment depression. Thirty nine of the 42 episodes of ST segment depression were preceded by arousal in which the electroencephalogram changed from a high amplitude slow wave pattern to a low amplitude high frequency pattern. This was followed immediately by an increase in heart rate from $68(10)$ beats a minute to $87(10)$ beats a minute, and only later did the ST segment start to change. All but one of the patients were awakened with chest pain at least once during each night of the study and were given sublingual glyceryl trinitrate.

Four episodes of ST segment depression were associated with rapid eye movement sleep periods. Two episodes followed increases in heart rate which
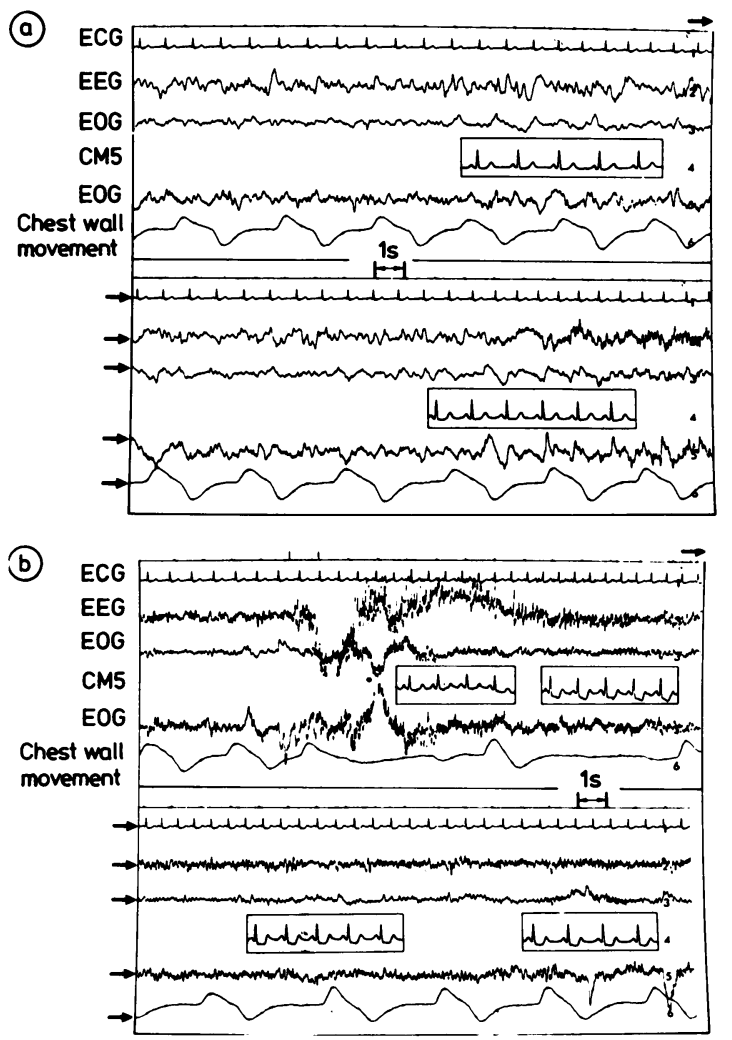

Fig 2 Polygraphic records ( $a$ and $b$ ) from a 63 year old woman with three vessel coronary artery disease during sleep demonstrating the onset of an episode of ST segment depression. The electroencephalogram (EEG), electrocardiogram (ECG), electro-ocluograms (EOG), and chest wall movements were recorded at $15 \mathrm{~mm} / \mathrm{s}$. Insets of the electrocardiogram recorded from lead CM5 at $25 \mathrm{~mm} / \mathrm{s}$ are shown. Sleep stage III followed by sudden arousal to stage I/II was associated with an increase in heart rate. Further increase in heart rate after turning (movement artefact) was followed by the onset of ST segment depression. 

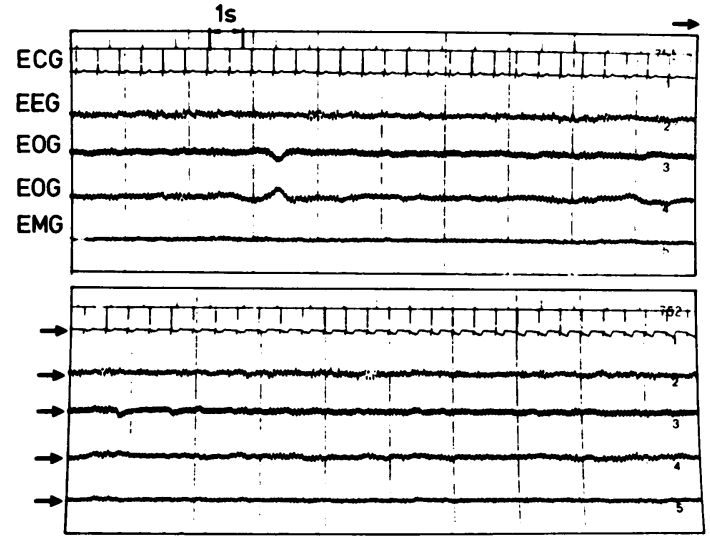

Fig 3 Polygraphic recording from a 58 year old woman with normal coronary arteries and spasm in the left circumflex coronary artery during sleep showing the onset of an episode of ST segment depression. The electrocardiogram (ECG), electroencephalogram (EEG), electro-oculogram (EOG), and electromyogram (EMG) were continuously recorded. There was no change in sleep stage or heart rate before the onset of $S T$ depression.

occurred during the later phases of rapid eye movement sleep. The other episodes occurred when arousal from rapid eye movement sleep to a lighter sleep stage occurred, again preceded by an increase in heart rate. Epoch marking of the electroencephalogram recordings throughout the night showed that all patients spent a proportionately greater time in lighter sleep stages $(1,2$, or awake) probably as a result of repeated awakening with chest pain. Rapid eye movement sleep, however, occurred in all patients, lasted for a mean of $44 \mathrm{~min}$ each night, and was more prevalent during the second night. On several occasions arousal from deeper sleep stages to lighter sleep occurred and was accompanied by an abrupt increase in heart rate but this was not followed by important ST segment depression $(>1 \mathrm{~mm}$ ) or pain. There was, however, a $<1 \mathrm{~mm}$ shift in the ST segment. In the absence of another independent marker it is impossible to know whether such changes also constitute ischaemia. In this study we have reported only those episodes of ST segment change that were $\geqslant 1 \mathrm{~mm}$, although it is likely that smaller changes also represent periods of less severe ischaemia. There were also differences in the baseline heart rate and the magnitude and rate of increase of the heart rate during different episodes of arousal in the same patient.

\section{Patients with coronary artery spasm}

Twenty three of the 26 episodes of ST segment change observed in the two patients with spasm were not preceded by increases in heart rate, changes in sleep stages, or bodily movement (fig 3 ). Episodes started with an initial change in the $T$ wave vector, progressed to peaking of the $T$ wave and important ST segment elevation, and ended with ST segment depression. There were 19 episodes of ST segment depression and seven episodes of ST segment elevation. In one patient, however, there were three episodes (our of a total of 14) of ST segment depression that were preceded by a lightening of sleep pattern, bodily movement, and increases in heart rate which resembled episodes in patients who had obstructive coronary artery disease.

\section{RELATION BETWEEN NOCTURNAL ST SEGMENT CHANGES AND RESPIRATION}

In one patient with three vessel coronary artery disease and episodes of ST segment depression at night, one episode was preceded by central apnoea with cessation of chest wall movements and nasal airflow for $22 \mathrm{~s}$. Oxygen saturation fell from a baseline of $96 \%$ to $82 \%$ and this was followed by an increase in heart rate and onset of ST segment depression (fig 4). None of the other episodes of ST segment depression or elevation, in this or any other patient, was preceded or accompanied by changes in respiratory pattern or oxygen saturation. This patient had other episodes of ST segment depression that were not preceded by apnoea but occurred as a result of lightening of sleep and bodily movements. He also had further brief episodes of central respiratory depression (fig 4) with reductions in chest wall movement and nasal airflow, but these did not result in a reduction of oxygen saturation or a change in heart rate or the ST segment.

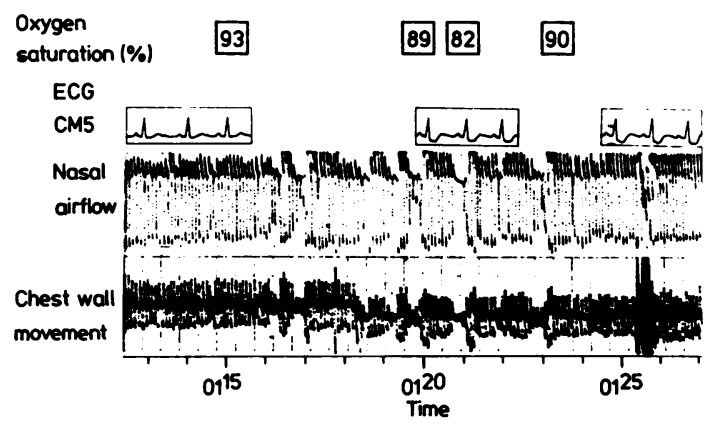

Fig 4 Recordings of the nasal airflow, chest wall movement $(25 \mathrm{~mm} / \mathrm{min})$, electrocardiogram $(25 \mathrm{~mm} / \mathrm{s})$, and oxygen saturation during sleep in a 68 year old man with three vessel coronary artery disease demonstrating several brief episodes of central apnoea with the final episode of apnoea lasting $22 \mathrm{~s}$. This was followed by desaturation, increase in heart rate, and $S T$ segment depression. 


\section{Discussion}

In this study we have examined two distinctly separate groups of patients who had frequent daytime and nocturnal angina pectoris. One group had obstructive coronary artery disease or positive exercise test at a low workload or both, whereas the other group had no important coronary artery disease and had negative exercise tests and localised coronary spasm. Most of the ischaemic episodes of ST segment depression in patients with coronary artery disease were preceded by movement, arousal, and increases in heart rate. We analysed the momentary changes of heart rate that preceded episodes of ST segment change occurring at night in a previous study. ${ }^{9}$ In those patients, all with severe obstructive coronary artery disease, almost all of the episodes of ST segment depression were preceded by small but significant increases in heart rate of the order of 20 (12) beats per minute. This study has demonstrated that these increases in heart rate are chiefly a result of arousal and lightening of the sleep phase or are associated with rapid eye movement sleep. Central apnoea occurred in only one subject and though this slightly reduced oxygen saturation (to $82 \%$ ), there was a sudden increase in heart rate, which possibly was more important in initiating myocardial ischaemia. At other times of the night further episodes of ST segment depression that followed bodily movements and arousal developed in this patient. The occurrence of this degree of sleep apnoea in the symptom free population is believed to be not uncommon, although the true incidence is unknown. ${ }^{11}$ To our knowledge there is no information on its relation with angina pectoris.

Whereas some studies have shown that episodes of nocturnal ST segment change occurred during the rapid eye movement phase of sleep, ${ }^{12}{ }^{13}$ others have reported no relation between rapid eye movement sleep and nocturnal angina. ${ }^{1415}$ Wide fluctuations of heart rate and blood pressure are also known to occur during rapid eye movement sleep as a result of autonomic stimulation. ${ }^{16}{ }^{17}$ In our study, episodes associated with rapid eye movement sleep were uncommon. Analysis of the sleep pattern confirmed that patients spent a proportion of their sleep time in the rapid eye movement phase of sleep. Because patients repeatedly awoke with chest pain several times each night the periods of rapid eye movement associated sleep were short, especially during the first night. We attempted to overcome this difficulty by having four patients sleep for two nights; they had longer periods of rapid eye movement sleep on the second night.

It was not the purpose of this study to establish the sleep, pattern of patients with nocturnal angina. This would have required several nights of acclimatisation in the sleep laboratory. This was not acceptable in our patients because they were suffering from frequent angina and were being investigated after withdrawal of all antianginal medication. Lightening of sleep, arousal, and bodily movements are normal during sleep and are followed by an increase in heart rate and blood pressure. ${ }^{1617}$ The magnitude of these haemodynamic changes varies between different episodes of arousal. In this study several episodes of arousal occurred that increased heart rate but were not followed by important ST segment depression $(>1 \mathrm{~mm})$. There may be several reasons for this. Firstly, we arbitrarily used $1 \mathrm{~mm}$ of ST segment depression as being representative of ischaemia. Often, episodes of heart rate increase were followed by depression of the ST segment that did not reach $1 \mathrm{~mm}$. These may have indicated periods of less severe myocardial ischaemia. Secondly, some episodes of arousal were followed by an increase in heart rate that was less than that which occurred when such arousal was accompanied by bodily movements such as turning. The latter was more likely to result in important ST segment depression. Also a slower arousal or bodily movements may result in a less rapid increase in heart rate which may also influence the development of ischaemia.

Finally, heart rate is just one of the determinants of myocardial oxygen demand. In this study we did not attempt to measure changes in blood pressure and end diastolic volume, which also influence myocardial oxygen demand. It is known that arousal and rapid eye movement sleep are also associated with fluctuations in blood pressure ${ }^{1617}$ and that recumbency increases end diastolic volume. If the magnitude of these changes varied during the course of the night it is not surprising that only some of the episodes of arousal were followed by important ST segment changes.

The size of the increase in heart rate before the onset of ST segment depression in patients with coronary artery disease varied in the same individual at different times of the night. The interval between the heart rate increase and the onset of ST segment depression also varied. This may have been either because differences in the magnitude of the stimulus were causing the change (arousal only versus arousal and turning) or because the magnitude of change in other factors such as blood pressure, end diastolic pressure, or coronary tone varied at different times of the night. Exercise tolerance in these patients was very low. We did not measure the heart rate at the onset of ST segment depression during the exercise tests. The resting heart rate and blood pressure during the daytime are higher than at night and it is therefore difficult to compare the absolute levels of 
heart rate or the magnitude of increase in heart rate during the exercise test with the increase that preceded ST segment changes at night.

It is impossible to exclude the influence of alterations in coronary tone, ${ }^{6}$ platelet aggregation, ${ }^{18}$ and other factors that may increase the obstruction of the coronary lumen in patients with severe coronary artery disease. Indeed there is evidence that coronary artery tone is higher during the night than during the day ${ }^{1920}$ and this may predispose patients to the development of ischaemia at lower levels of exertion at night than during the day. The important finding in this study is that in patients with severe coronary artery disease increases in myocardial oxygen demand immediately precede the onset of ischaemia at night as well as during the day.

Continuous haemodynamic monitoring in patients with variant angina has shown that no important changes in heart rate, blood pressure, left ventricular filling pressure, or cardiac output precede the onset of ST segment changes. ${ }^{67}$ In a proportion of patients in whom all night polygraphic studies were performed, ST segment elevation or depression was not associated with rapid eye movement sleep or with any changes in sleep pattern. ${ }^{12-14}$ In the present study episodes of ST segment change developed in two patients with proven coronary artery spasm; most were not preceded by changes in the heart rate, respiratory rate, or oxygen saturation. Episodes were more common during the early morning hours ${ }^{19}$ (fig 1 ). The factors that precipitate coronary artery spasm remain obscure. Coronary artery tone is increased at night and tends to be highest in the early morning hours ${ }^{1920}$; this may account for the increased frequency. Although all the episodes in one patient and 11 of the 14 episodes in the other patient were not preceded by increases in heart rate, three episodes of ST segment depression followed arousal and tachycardia. Possibly coronary spasm was partly but not completely occluding the vessel at these times and was not producing myocardial ischaemia under resting conditions. After arousal and an increase in heart rate, myocardial oxygen demand increased and this resulted in ischaemia and onset of ST segment depression.

Most of the episodes of ST segment elevation and depression were not accompanied by chest pain. In individual patients, however, the factors associated with and preceding the onset of painful and pain free episodes of ST segment change were identical. Similarities in haemodynamic and functional variables accompanying painless and painful episodes of ST segment change have been demonstrated before. ${ }^{21}$ The factors that precipitate myocardial ischaemia at rest in patients with obstructive coronary artery dis- ease and limited coronary reserve differ from those that precipitate ischaemia in patients with normal coronary arteries and spasm. The importance of increased myocardial oxygen demand in the former group of patients has been clearly demonstrated and its therapeutic implications need to be further investigated.

We thank the British Heart Foundation for support and $\mathrm{Dr} \mathrm{T}$ McMorland for his assistance.

\section{References}

1 Heberden W. Some account of disorder of the breast. Medical Transactions of the College of Physicians of London 1772;2:59-67.

2 Osler W. The Lumelian lectures on angina pectoris. Lancet 1910;i:697-702.

3 Prinzmetal M, Ekmecki A, Kennamer R, et al. Variant form of angina pectoris: previously undelineated syndrome. $\mathcal{F} A M A 1960 ; 174: 1791-800$.

4 MacAlpin RN, Kattus AA, Alvaro AB. Angina pectoris at rest with preservation of exercise capacity: Prinzmetal's variant angina. Circulation 1973;47:476-85.

5 Chierchia S, Gallino A, Smith G, et al. Role of heart rate in pathophysiology of chronic stable angina. Lancet 1984;ii:1353-7.

6 Maseri A, Severi S, De Nes M, et al. 'Variant' angina: one aspect of a continuous spectrum of vasospastic myocardial ischemia: pathogenetic mechanisms, estimated incidence and clinical and coronary arteriographic findings in 138 patients. Am $\mathcal{f}$ Cardiol 1978; 42:1019-35.

7 Figueras J, Singh BN, Ganz W, Charuzi Y, Swan HJC. Mechanisms of rest and nocturnal angina: observations during continuous hemodynamic and electrocardiographic monitoring. Circulation 1979;59: 955-68.

8 Schang SJ, Pepine CJ. Transient asymptomatic ST segment depression during daily activity. Am $\mathcal{F}$ Cardiol 1977;39:396-402.

9 Quyyumi AA, Wright CA, Mockus LJ, Fox KM. Mechanisms of nocturnal angina pectoris: the importance of increased myocardial oxygen demand in patients with severe coronary artery disease. Lancet 1984;i:1207-9.

10 Rechtschaffen A, Kales A, eds. A manual of standardised terminology, techniques and scoring system for sleep stages of human subjects. Washington DC: Government Printing Office, 1968.

11 Block AG, Boysen PG, Wynne JW, Hunt LA. Sleep apnea, hypopnea and oxygen desaturation in normal subjects: a strong male dominance. $N$ Engl $\mathfrak{f} \mathrm{Med}$ 1979;300:513-7.

12 Murao S, Harumi K, Katayama S, et al. All-night polygraphic studies of nocturnal angina pectoris. $f p n$ Heart $\mathcal{F}$ 1972;13:295-306.

13 Nowlin JB, Troyer WG Jr, Collins WS, et al. The association of nocturnal angina pectoris with dreaming. Ann Intern Med 1965;63:1040-6.

14 Chierchia S, Guazzelli M, Maggini C, Maseri A. 
Absence of correlation of nocturnal angina with sleep stages [Abstract]. Circulation 1978;58 (suppl ii):194.

15 Faracon I, Williams RL, Taylor WJ. Sleep characteristics of patients with angina pectoris. Psychosomatics 1969;10:280-4.

16 Khatri IM, Freis ED. Hemodynamic changes during sleep. F Appl Physiol 1967;22:867-73.

17 Snyder F, Hobson JA, Morrison DF, Goldfrank F. Changes in respiration, heart rate and systolic blood pressure in human sleep. f Appl Physiol 1964; 19:417-22.

18 Folts JD, Crowell JREB, Rowe GG. Platelet aggregation in partially obstructed vessels and its elimination with aspirin. Circulation 1976;54:365-70.
19 Araki H, Koiwaya Y, Nakagaki O, Nakamura $M$. Diurnal distribution of ST-segment elevation and related arrhythmias in patients with variant angina: a study by ambulatory ECG monitoring. Circulation 1983;67:995-1000.

20 Yasue H, Omote S, Takizawa A, Nagao H, Miwa K, Tanaka S. Circadian variation of exercise capacity in patients with Prinzmetal's variant angina: role of exercise-induced coronary arterial spasm. Circulation 1979;59:938-48.

21 Chierchia S, Lazzari M, Freedman B, Brunelli C, Maseri A. Impairment of myocardial perfusion and function during painless myocardial ischemia. $7 \mathrm{Am}$ Coll Cardiol 1983;1:926-30. 